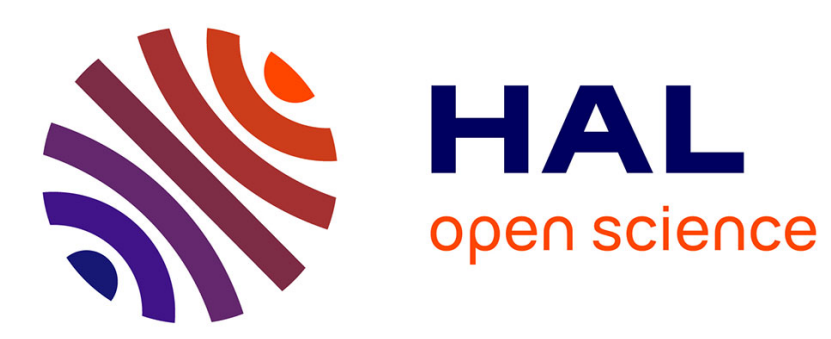

\title{
Determining radius and position of a sphere from a single catadioptric image
}

Simone Gasparini, Vincenzo Caglioti

\section{To cite this version:}

Simone Gasparini, Vincenzo Caglioti. Determining radius and position of a sphere from a single catadioptric image. Journal of Intelligent and Robotic Systems, 2008, 52 (3-4), pp.447-463. 10.1007/s10846-008-9225-6 . hal-03389139

\section{HAL Id: hal-03389139 \\ https://hal.science/hal-03389139}

Submitted on 20 Oct 2021

HAL is a multi-disciplinary open access archive for the deposit and dissemination of scientific research documents, whether they are published or not. The documents may come from teaching and research institutions in France or abroad, or from public or private research centers.
L'archive ouverte pluridisciplinaire HAL, est destinée au dépôt et à la diffusion de documents scientifiques de niveau recherche, publiés ou non, émanant des établissements d'enseignement et de recherche français ou étrangers, des laboratoires publics ou privés. 


\title{
Determining radius and position of a sphere from a single catadioptric image
}

\author{
Vincenzo Caglioti and Simone Gasparini \\ Dipartimento di Elettronica e Informazione, \\ Politecnico di Milano \\ Piazza Leonardo da Vinci, 32 \\ 20133 Milano (Italy) \\ \{caglioti,gasparini\}@elet.polimi.it
}

\begin{abstract}
In order to improve robot capabilities related to playing with a flying ball, reliable methods to localize a sphere in the 3D space are needed. When the radius of the sphere is known, it can be localized by analyzing a single, perspective image of it. When the sphere radius is not known, a single perspective image is not sufficient. In this paper we consider axial-symmetric catadioptric cameras, i.e. devices consisting of an axial-symmetric mirror plus a perspective camera, whose viewpoint is on the symmetry axis. If the viewing rays are not all concurrent at a single point, this camera is said to be non-central. We show that, using a noncentral axial-symmetric catadioptric camera, a single image is sufficient to determine both the position of a sphere and its radius. Some preliminary experimental results are also presented.
\end{abstract}

\section{Introduction}

The recovery of 3D information from 2D images can find many applications both in Computer Vision and in Mobile Robotics. In this work we deal with the determination of both the radius and the 3D position of a sphere from a single image. Using a standard perspective camera, if the radius is known, a sphere can be localized from its apparent contour image. Consider a sphere of radius $R$ and let $D$ be the (unknown) distance between the sphere center and the camera viewpoint. Consider now the two points of the contour image of the sphere for which the angle between the associated viewing rays is maximum: if $\alpha$ is the semi-angle between these two rays, then it yields that $\sin \alpha=R / D$, which can be solved to determine $D$. Therefore the sphere can be easily localized.

This task becomes challenging when no a priori information about the sphere is given. To tackle this problem we use a so-called catadioptric camera, which is generally constituted by a mirror placed in front of a perspective camera. We show that under broad conditions we can determine both the radius and position of a sphere from a single catadioptric image.

The main application of this work is in the field of mobile robotics, where the localization of a sphere, i.e. a ball, is a crucial task for robots playing soccer in RoboCup contests [8]. In order to detect and localize a ball, many approaches 
has been employed. In [15] a stereo vision system is used to give an optimal estimation of the 3D position of the ball and the other objects in the field of play. The approach with single frontal perspective camera is also widely exploited: the most common techniques for ball detection rely on color information and are based on fast color segmentation of the image to detect and track objects [3], [5]. Recently a color-independent technique has been presented by [13] which developed an edge-based ball detection system with an Ada Boost learning procedure that constructs a cascade of classification and regression trees. Also the system proposed by [7] is a color independent edge tracking algorithm and allows to locate arcs and circle in order to identify the ball. Catadioptric cameras are also used since they enlarge the field of view of the camera and allow the robot to see all the objects around [12]. However, for all these methods the 3D localization of the ball can not be computed without knowing the radius of the ball (the size of the ball is standardized in each RoboCup League), or making other assumptions, such that all relevant objects are located on the ground of the field [3], or by using team coordination methods in order to integrate in a SLAM framework the sensing information coming from other teammates [18].

Spherical objects have been also investigated for calibration purpose: Agrawal et al. [1] and Zhang [20] used image of spheres to calibrate (frontal) perspective cameras while Yingv [19] developed a calibration procedure for central catadioptric cameras using the occluding contour of a sphere in the catadioptric image.

This paper is structured as follows: in Section 2 catadioptric cameras and some related basic geometric properties are introduced, and the addressed problem is formulated. In Section 3 the geometric aspects of the determination of radius and distance of a sphere with a non-central axial symmetric catadioptric camera are discussed and in Section 4 the resolution algorithm is described. In Section 5 some preliminary experimental results are presented both with synthetic and real images. Section 6 concludes the paper.

\section{Catadioptric cameras and problem formulation}

A catadioptric camera is generally constituted by a curved mirror placed in front of a perspective camera. In a catadioptric camera, the viewing ray coming from a scene point is specularly reflected by the mirror surface, before it goes through the camera viewpoint and crosses the image plane. Catadioptric cameras are attractive because of the possibility to employ them in omni-directional vision.

A catadioptric camera is calibrated, if the viewing ray associated to each image point is known.

If all viewing rays concur at a same point (called "center"), the camera is said to be central. A central camera can be obtained by placing a hyperbolic mirror, or an elliptical mirror, in front of a perspective camera, in such a way that the viewpoint of the camera is on one of the foci of the mirror surface [2]. In this way, the center, where all viewing rays concur, is on the other focus (Figure 1).

Any calibrated central camera reduces to a perspective camera. 


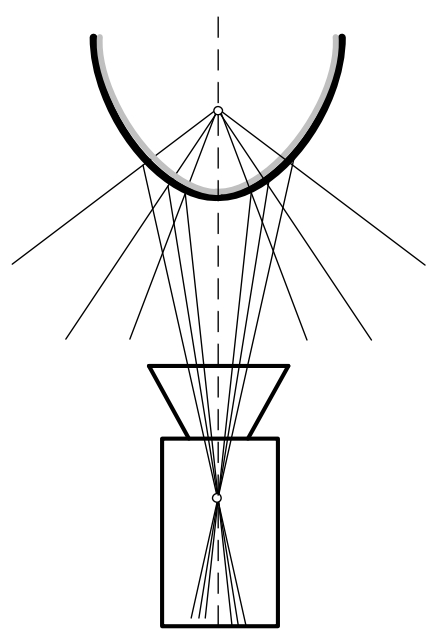

(a)

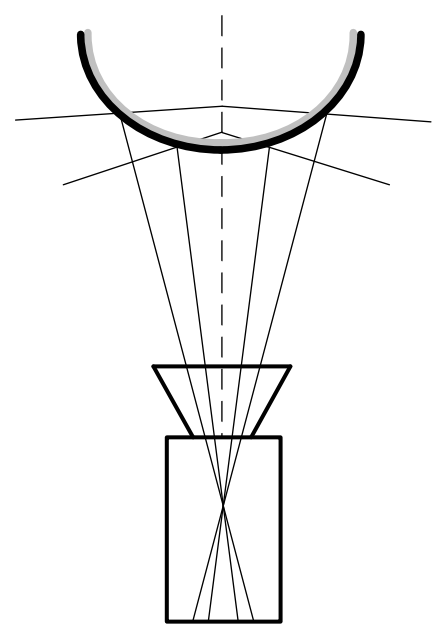

(b)

Fig. 1. Examples of catadioptric cameras: (a) a central catadioptric camera based on a paraboloidal mirror in which the camera viewpoint is placed on one of the foci of the mirror so that all the viewing rays concur at the other focus ; (b) in a noncentral catadioptric camera based on spherical mirror the viewing rays do not concur at the same point.

Noncentral cameras, cameras whose viewing rays are not all concurrent [16], can be realized, e.g., by linear push-broom cameras [10], cross-slit cameras [9], catadioptric cameras [14].

With respect to central cameras, noncentral cameras remove some degeneracies: e.g., the viewing surface, i.e., the union of the viewing rays, of a straight line in the 3D space is not planar. This fact has can be exploited to localize a straight line in the $3 \mathrm{D}$ space from a single image [6].

On the other hand, some difficulties arise in the image-based characterization of curved (self-occluding) surfaces. In particular, using a central camera, the plane tangent to a curved surface at a point on the contour generator can easily be derived from the image: it is the backprojection of the tangent to the apparent contour. Using a noncentral camera, since the viewing rays can be skew, the image-based characterization of the plane tangent to a curved surface is more difficult.

In the sequel, we will focus on single-image cameras, i.e., cameras such that through any space point external to the mirror surface there is only one viewing ray. It can be shown that if the mirror surface is convex the camera is singleimage.

According to the specular reflection laws, the viewing ray associated to an image point $p$, is the line $l$ through the point $B$ (where the line $O p$ intersects the mirror surface) such that the normal to the mirror surface at $B$ bisects the angle between $O B$ and $l$. 


\subsection{Axial-symmetric catadioptric cameras}

Now, axial-symmetric catadioptric cameras are considered.

An axial-symmetric catadioptric camera consists of an axial-symmetric mirror and a perspective camera, whose viewpoint $O$ is on the symmetry axis of the mirror.

The mirror surface of an axial-symmetric catadioptric camera can be obtained by rotating a planar curve, called profile, about the symmetry axis. The intersection between the mirror surface and the symmetry axis is called apex.

Notice that, since the symmetry axis of the mirror goes through the viewpoint of the perspective camera, the image (by means of the perspective camera) of the symmetry axis collapses into a single point. This condition is not related to placing the camera such that the image of the apex coincides with the principal point.

Planes containing the symmetry axis of the mirror are said to be axial planes. From symmetry, the viewing ray through any space point $P$ must be contained in the axial plane through $P$ : therefore, all the viewing rays cross the symmetry axis (possibly, at the infinite).

By symmetry, any viewing ray is coplanar with the $z$-axis: therefore each viewing ray crosses the $z$ axis at a point (possibly, at the infinite).

By rotational symmetry, the set of all the viewing rays can be subdivided into "equivalence classes", where all rays in a same equivalence class form the same angle with the symmetry axis, and cross it at a same point. Each equivalence class identifies a (right) cone of rays, whose axis is the symmetry axis.

\subsection{Problem formulation}

The addressed problem is formulated. A calibrated, axial-symmetric catadioptric camera is given. In a calibrated camera, the mapping between any pixel and its associated viewing ray is known.

The mirror surface is supposed to be convex, so that any point in the free space is crossed by only one viewing ray: i.e., the camera is single-image.

A sphere of unknown radius is placed at an unknown position in the 3D space. The sphere is entirely visible from the catadioptric camera, namely, it is only occluded by itself.

The apparent contour of an unknown sphere is extracted.

The addressed problem is to determine both the radius and the position of the sphere, starting from its apparent contour.

\section{On the apparent contour of a sphere viewed by a noncentral axial-symmetric camera}

The proposed method for determining radius and position of the sphere uses the following relevant features: a specific pair of viewing rays, and a specific pair of axial planes. 
A. A specific pair of viewing rays.

By placing a sphere at a generic position relative to a noncentral, axialsymmetric camera, axial-symmetry is removed. However, a planar symmetry is preserved: the system constituted by the axial-symmetric camera plus the sphere is symmetric about the plane, which contains the sphere center and the camera (mirror) axis.

This symmetry plane is an axial plane.

Since this plane is a symmetry plane for the sphere, it cuts the sphere at a great circle $C$. The circumference of this great circle is said to be a "meridian" of the sphere. Hence the viewing rays, contained in the symmetry plane, which are tangent to the sphere, are tangent to the meridian $C$.

Since the sphere is visible, then there are (at least) two such tangent rays within the symmetry plane. In general, from mirror convexity, there are only two tangent rays within the symmetry plane. The two rays of this pair intersect at a point $B$ within the symmetry plane: in general this intersection point does not lie on the mirror axis.

B. A specific pair of axial planes.

Consider the two axial planes tangent to the sphere: these planes are bisected by the symmetry plane. Also the two tangency points are symmetric w.r.t. the symmetry plane. The two viewing rays through the tangency points are tangent to the sphere, since they are contained in the tangent planes. These two viewing rays are symmetric w.r.t. the symmetry plane as well.

The two tangency points belong to a same great circle (see Figure 2). This great circle is symmetric w.r.t. the symmetry plane, and it is perpendicular to the direction of the mirror axis: we call this great circle the "equator" of the sphere, and we indicate it by $E$.

\section{Determining radius and position of the sphere}

First we consider the two axial planes tangent to the sphere. Let $A$ be the image of the mirror axis: as said in Section 2.1, this image is a single point.

Image lines through this point $A$ backproject onto axial planes. Therefore, the two (most angularly separated) image lines through $A$, that are tangent to the apparent contour of the sphere, backproject to the axial planes that are tangent to the sphere (see Figure 2).

From calibration, the angle formed by these tangent planes can be determined from the image lines tangent to the apparent contour of the sphere.

Let $\alpha$ be the semi-angle formed by these two tangent planes. Let $D$ be the unknown distance between the sphere center and the mirror axis, and let $R$ be the unknown radius of the sphere:

$$
\frac{R}{D}=\sin \alpha
$$

Now the two viewing rays, tangent to a meridian of the sphere, are determined. 


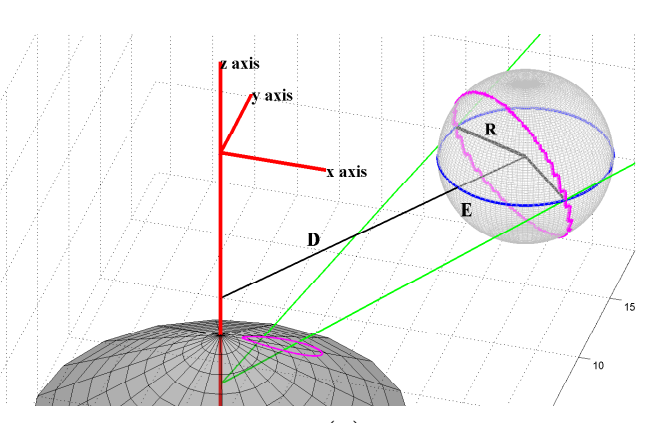

(a)

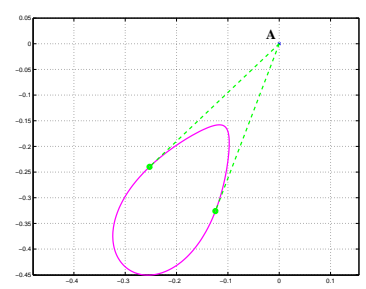

(b)

Fig. 2. Example of rays tangent to a great circle of the sphere in a non-central axial symmetric catadioptric camera: (a) the $3 \mathrm{D}$ model with the camera placed at the origin of the axes looking at the conical mirror in gray (bottom) and the apparent contour of the sphere reflected on the mirror (points in magenta); (b) the image points of the apparent contour of the sphere. The green rays in (a) are the viewing rays associated to the two tangent points of image contour (circled in green in (b)) w.r.t. the image point $A$ of the mirror axis: these rays are tangent to the sphere and to the great circle $E$ on the sphere itself (in blue).

The symmetry plane is the plane bisecting the two above axial planes tangent to the sphere. From calibration, one can determine the image projection of the symmetry plane: this projection is again a line through $A$. The two intersection points between this image line and the apparent contour correspond to two viewing rays that are both tangent to the sphere, and contained in the symmetry plane. From the argument in the previous subsection, these viewing rays are tangent to the meridian $C$ of the sphere. These viewing rays are completely characterized from calibration. Let $l$ be the line bisecting these two viewing rays, and let $\beta$ be the angle formed by $l$ and either of them. Let $\gamma$ be the angle formed by $l$ and the direction of the mirror axis. Let $\Delta$ be the distance between the mirror axis and the intersection point $B$ of the two rays.

From Figure 3, we derive:

$$
\frac{R}{D-\Delta}=\frac{\sin \beta}{\sin \gamma}
$$

Angle $\beta$ can be calculated in two equivalent ways:

1. Consider the two contour points whose viewing rays are both contained in the symmetry plane and calculate the semi-angle between these two rays;

2. Consider the two contour points for which the angle between the associated viewing ray and the $z$-axis is maximun and minimum respectively, and calculate the semi-angle between these two rays;

The two methods are geometrically equivalent, but the second method appears to be more robust to noise. 


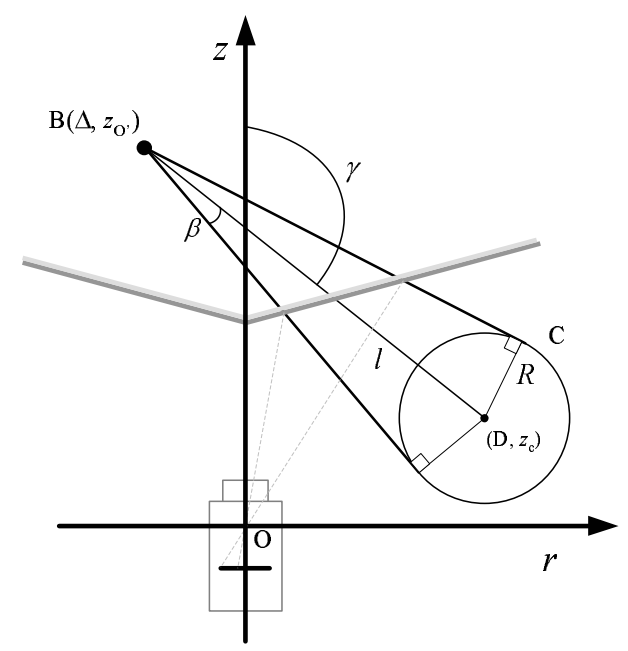

Fig. 3. An example of rays tangent to a meridian of a sphere in a non-central axial symmetric catadioptric camera with a conical mirror; the coordinate system $(r, z)$ (assuming $r=\sqrt{x^{2}+y^{2}}$ ) is referred to the symmetry plane containing the $z$-axis and passing through the center of the sphere.

Since the angles $\alpha, \beta$ and $\gamma$ are known from the image and from calibration, the two last equations can be solved for the two unknowns $R$ and $D$. In this way both the radius $R$ of the sphere, and its distance $D$ from the mirror axis are determined. The localization can be completed by using the line $l$ bisecting the two rays, which are tangent to the sphere at the meridian $C$. The sphere is centered at the point of $l$, whose distance from the mirror axis is $D$.

The localization is completed.

\section{$5 \quad$ Preliminary experimental results}

In order to validate the above method we performed some experiments both on synthetic data and real images.

\subsection{Experiments with synthetic data}

We developed a 3D simulator for a catadioptric camera based on a conical mirror. Given the position and the size of a sphere, the relevant apparent contour image is generated. Then two pairs of symmetric points are chosen, namely the pair of tangent points w.r.t. the axis image point $\mathrm{A}$, and the pair of points lying on the symmetry axis of the contour. For each pair the relevant viewing rays is calculated in order to solve the system of Equation 1 and Equation 2.

We performed some experiments using synthetic image rendered by POV-Ray [17], an open-source rendering engine. We model the mirror as a high-reflective 
cone placed in front of the (virtual) camera with its symmetry axis lying on the optical axis of the camera. Then we place some spheres of different size $R$ at various distances $D$ from the mirror axis. Figure 5.1 collects the images used in the experiments and the relevant value for $R$ and $D$. Once the catadioptric image is rendered, the image contour of the ball is extracted. Since the shape and the position of the cone w.r.t. the camera is known from the 3D model as well as the camera parameters, the $3 \mathrm{D}$ localization and radius estimate is then calculated by applying the algorithm explained in Section 4 and solving the system of Equation 1 and Equation 2.

In order to verify the robustness of the method we corrupted the previous rendered image with noise: for each image, Gaussian white noises with an increasing value of $\sigma$ was added. Figure 5 shows a detail of a image rendered with POV-Ray and the same detail corrupted with different Gaussian white noises.

Table 1 collects the results and the estimate errors. Table 1.a, which collects the results for images without noise, shows that the errors are smaller (less than $1 \%$ ) as the ball is placed near the mirror axis (Images 1 to 5), with the exception of Image 6 where the image of the ball is too small so that the whole algorithm is more prone to errors in detecting and selecting the pairs of points used to estimate both radius and distance. This leads to a larger localization error (about $2 \%$ on $D$ ). As the ball is placed far away from the mirror axis (Images 7 to 9 ) the errors increase (up to $5 \%$ on $D$ ). However the $R / D$ ratios are preserved since the $R / D$ errors are comparable to the errors of the previous images.

Tables 1.b-d show how noise affects the estimate of $R$ and $D$ as the $\sigma$ increase. In particular for a given distance $D$ the noise obviously affects the estimate of the ball with lower radius $R$. The worst case is the one of image 9 with an error of nearly $9 \%$ on $D$. However it should be noted that the error on $R / D$ ratios are less than $1 \%$ for all images. 
(a) $\sigma=0$

\begin{tabular}{cccccccc}
\hline Image & $R$ & $R / D$ & $R \hat{/ D}$ & $e_{R / D}(\%)$ & $D$ & $\hat{D}$ & $e_{D}(\%)$ \\
\hline 1 & 3 & 0.300 & 0.300 & 0.008 & 10 & 9.946 & 0.545 \\
2 & 2 & 0.200 & 0.200 & 0.117 & 10 & 9.935 & 0.646 \\
3 & 1 & 0.100 & 0.100 & 0.096 & 10 & 9.885 & 1.155 \\
4 & 5 & 0.263 & 0.263 & 0.037 & 19 & 18.906 & 0.497 \\
5 & 2.5 & 0.132 & 0.131 & 0.092 & 19 & 18.861 & 0.733 \\
6 & 1 & 0.053 & 0.052 & 0.378 & 19 & 18.595 & 2.131 \\
7 & 10 & 0.250 & 0.250 & 0.017 & 40 & 37.889 & 5.277 \\
8 & 7.5 & 0.188 & 0.187 & 0.028 & 40 & 39.145 & 2.137 \\
9 & 5 & 0.125 & 0.125 & 0.046 & 40 & 38.783 & 3.043 \\
\hline
\end{tabular}

(b) $\sigma=5$

\begin{tabular}{cccccccc}
\hline Image & $R$ & $R / D$ & $R \hat{/ D}$ & $e_{R / D}(\%)$ & $D$ & $\hat{D}$ & $e_{D}(\%)$ \\
\hline 1 & 3 & 0.300 & 0.300 & 0.004 & 10 & 9.946 & 0.539 \\
2 & 2 & 0.200 & 0.200 & 0.120 & 10 & 9.934 & 0.664 \\
3 & 1 & 0.100 & 0.100 & 0.113 & 10 & 9.881 & 1.192 \\
4 & 5 & 0.263 & 0.263 & 0.037 & 19 & 18.905 & 0.499 \\
5 & 2.5 & 0.132 & 0.132 & 0.172 & 19 & 18.711 & 1.522 \\
6 & 1 & 0.053 & 0.053 & 0.432 & 19 & 18.201 & 4.204 \\
7 & 10 & 0.250 & 0.251 & 0.463 & 40 & 37.036 & 7.411 \\
8 & 7.5 & 0.188 & 0.189 & 0.781 & 40 & 36.915 & 7.713 \\
9 & 5 & 0.125 & 0.126 & 0.789 & 40 & 36.384 & 9.041 \\
\hline
\end{tabular}

(c) $\sigma=10$

\begin{tabular}{cccccccc}
\hline Image & $R$ & $R / D$ & $R \hat{/ D}$ & $e_{R / D}(\%)$ & $D$ & $\hat{D}$ & $e_{D}(\%)$ \\
\hline 1 & 3 & 0.300 & 0.300 & 0.011 & 10 & 9.944 & 0.561 \\
2 & 2 & 0.200 & 0.200 & 0.130 & 10 & 9.930 & 0.699 \\
3 & 1 & 0.100 & 0.100 & 0.132 & 10 & 9.877 & 1.230 \\
4 & 5 & 0.263 & 0.263 & 0.022 & 19 & 18.888 & 0.588 \\
5 & 2.5 & 0.132 & 0.132 & 0.189 & 19 & 18.691 & 1.626 \\
6 & 1 & 0.053 & 0.053 & 0.462 & 19 & 18.194 & 4.242 \\
7 & 10 & 0.250 & 0.251 & 0.472 & 40 & 37.077 & 7.308 \\
8 & 7.5 & 0.188 & 0.189 & 0.791 & 40 & 36.945 & 7.638 \\
9 & 5 & 0.125 & 0.126 & 0.803 & 40 & 36.348 & 9.130 \\
\hline
\end{tabular}

(d) $\sigma=20$

\begin{tabular}{cccccccc}
\hline Image & $R$ & $R / D$ & $R \hat{\jmath} D$ & $e_{R / D}(\%)$ & $D$ & $\hat{D}$ & $e_{D}(\%)$ \\
\hline 1 & 3 & 0.300 & 0.300 & 0.058 & 10 & 9.935 & 0.648 \\
2 & 2 & 0.200 & 0.200 & 0.164 & 10 & 9.920 & 0.798 \\
3 & 1 & 0.100 & 0.100 & 0.191 & 10 & 9.860 & 1.398 \\
4 & 5 & 0.263 & 0.264 & 0.367 & 19 & 18.048 & 5.009 \\
5 & 2.5 & 0.132 & 0.132 & 0.246 & 19 & 18.635 & 1.921 \\
6 & 1 & 0.053 & 0.053 & 0.497 & 19 & 18.137 & 4.545 \\
7 & 10 & 0.250 & 0.251 & 0.447 & 40 & 36.968 & 7.580 \\
8 & 7.5 & 0.188 & 0.189 & 0.782 & 40 & 36.982 & 7.546 \\
9 & 5 & 0.125 & 0.126 & 0.901 & 40 & 36.040 & 9.900 \\
\hline
\end{tabular}

Table 1. Localization results for images rendered with POV-Ray. $R / D$ and $D$ is the real radius-distance ratio and the real distance value (in $\mathrm{cm}$ ); $R \hat{/ D}$ and $\hat{D}$ is the estimated ratio and distance (in $\mathrm{cm}) . e_{R / D}(\%)$ and $e_{D}(\%)$ are the percentage estimation error. 


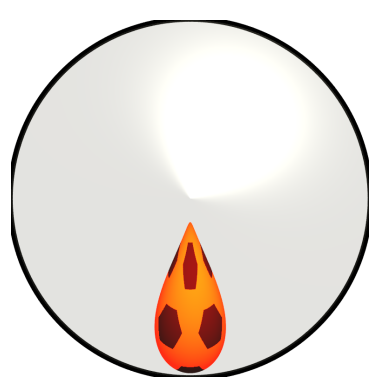

(1) $R=3 D=10$

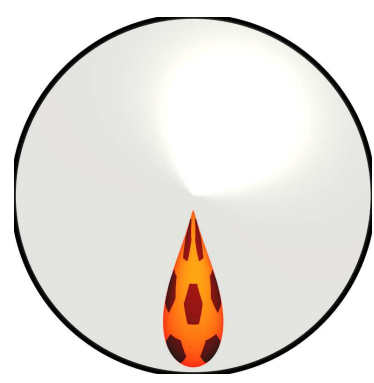

(4) $R=5 D=19$

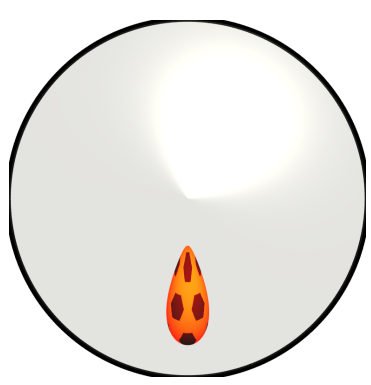

(2) $R=2 D=10$

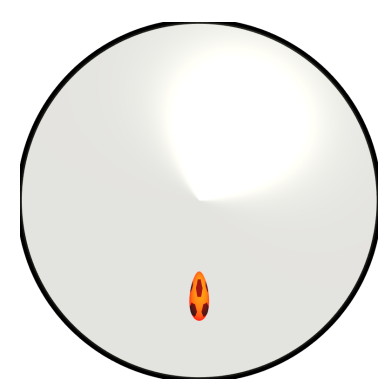

(3) $R=1 \quad D=10$

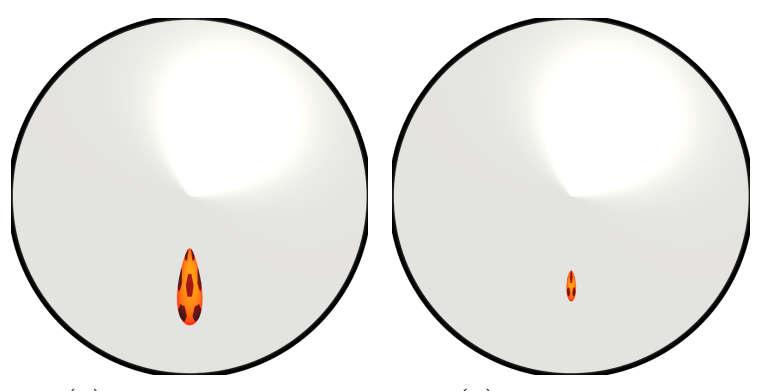

(6) $R=1 D=19$

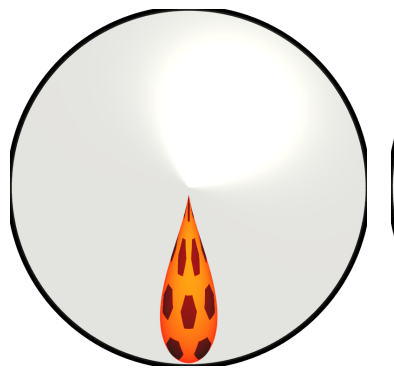

(7) $R=10 D=40$

(5) $R=2.5 D=19$

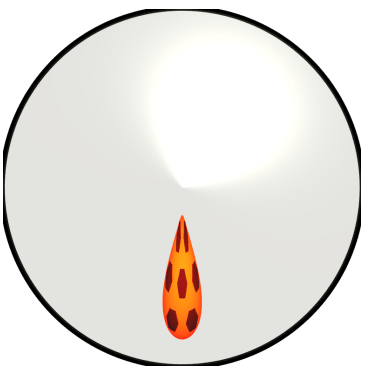

(8) $R=7.5 D=40$

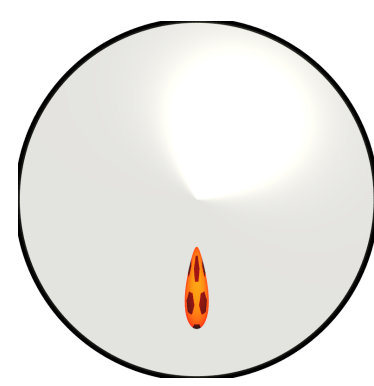

(9) $R=5 D=40$

Fig. 4. The set of rendered images used in our experimental activities. The mirror has been modeled as a highly-reflective cone with a semi-aperture of $45^{\circ}$ and radius of $5 \mathrm{~cm}$. The cone is placed in front of the (virtual) camera such that the symmetry axis of the mirror and the optical axis coincide; the ball has been modeled as a sphere of radius varying from $1 \mathrm{~cm}$ to $10 \mathrm{~cm}$ and placed at a distance varying from $10 \mathrm{~cm}$ to $40 \mathrm{~cm}$ far from the mirror axis. 


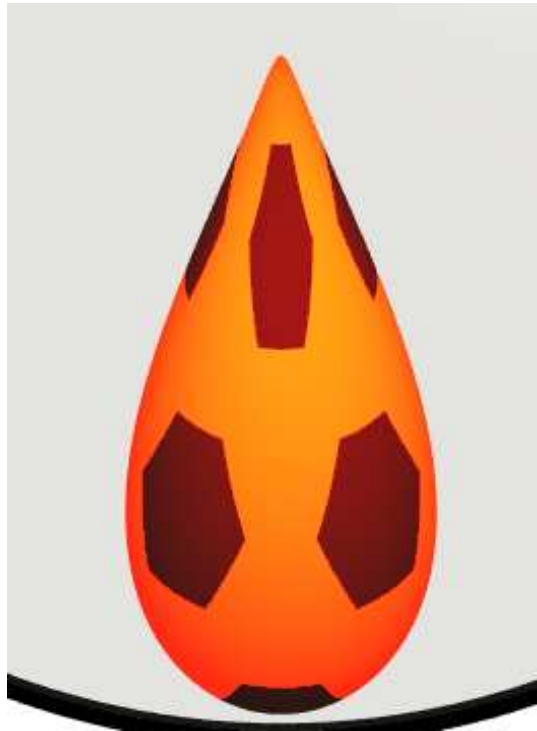

(a) $\sigma=0$

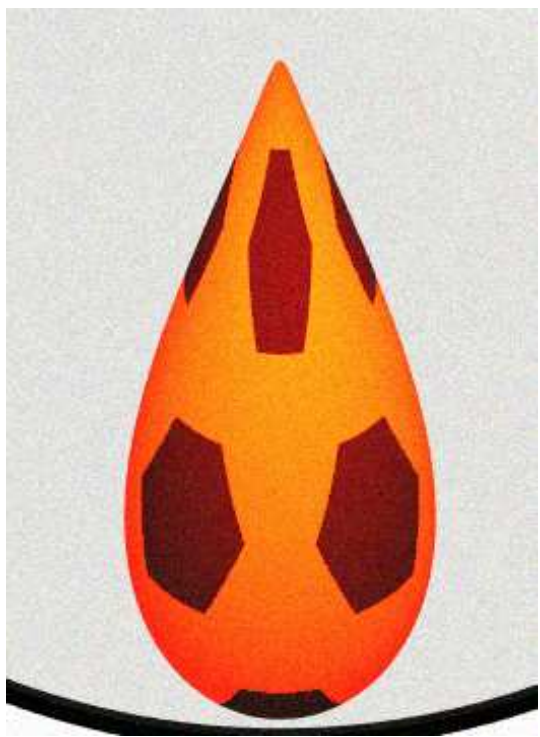

(c) $\sigma=10$

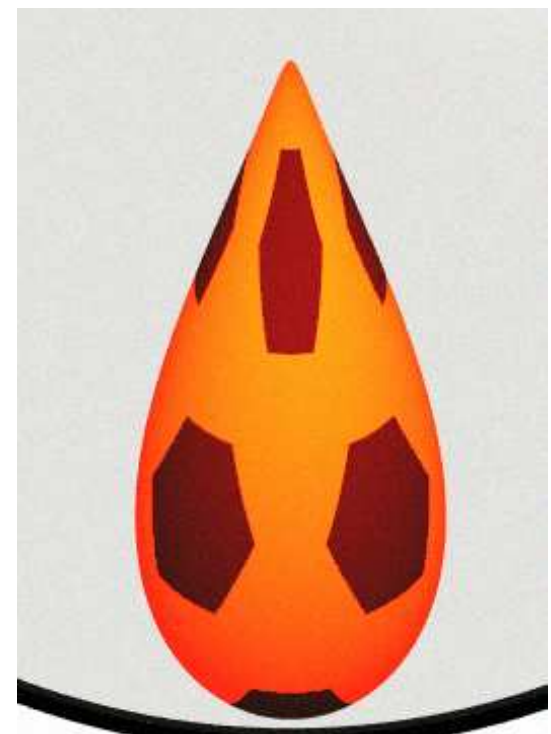

(b) $\sigma=5$

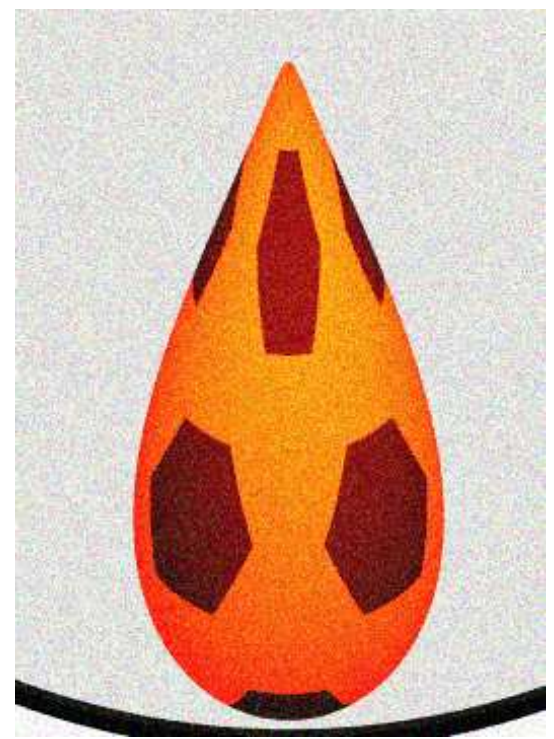

(d) $\sigma=20$

Fig. 5. A detailed view of Image 1 (a) and the same detail corrupted with an additive Gaussian white noise with $\sigma=5$ (b), $\sigma=10$ (c) and $\sigma=20$ (d). 


\subsection{Experiments with real images}

We also performed some preliminary experiments with real images. As a case study we chose a catadioptric camera with a conical mirror. However it is important to remark that the above method can be applied to any noncentral, axial symmetric catadioptric camera whose mirror is convex. We analyzed some images with a soccer ball, similar to the one used in some Robocup Leagues, and with a yellow tennis ball. The image contours are extracted and the estimated radii and distances are compared with the ground truth.

In this preliminary experimentations, we followed a rough two-step calibration method: first, the intrinsic camera parameters [21] is estimated using the Matlab Calibration Toolbox [4]. Then the external circumference of the conical mirror is localized w.r.t. the camera following the method described in [11].

The image of the ball is detected using color information: we develop a colorbased detector inspired to [5] that automatically detect the ball image. In order to extract the apparent contour of the sphere we have developed a fast sub-pixel algorithm for edge extraction based on Gaussian interpolation, which allows to reach an accuracy of one twentieth of a pixel. Once the apparent contour points are extracted we applied the procedure described in Section 4.

Figure 6 shows two sample catadioptric images with a tennis ball, while Figure 7 shows two sample catadioptric images with a soccer ball. Table 2 reports the results for each image with the relevant errors in estimating the radius and the distance of the ball from the mirror axis. The percentage errors are in the most cases less than $10 \%$. We think that the errors are due to the rough calibration of the whole system and to an incorrect alignment of the camera and the mirror, which yields the image of the symmetry axis not to collapse into a single image point. However we are developing a more reliable and robust calibration procedure in order to minimize the misalignment of the camera and thus to reduce errors.

In order to make the procedure more robust we are also planning to develop an optimization method which, starting from the estimates calculated solving Equation 1 and Equation 2, considers even the other contour points in order to minimize, for example, the distance error from the estimated center of the sphere.

\section{Conclusions}

A study has been presented on the determination of radius and position of a sphere in 3D space from single 2D images, acquired by a catadioptric camera constituted by an axial symmetric mirror plus a perspective camera with the viewpoint lying on the symmetry axis of the mirror. The geometric aspects of catadioptric cameras are presented and a simple procedure for determining the distance and the radius of the sphere from its occluding image contour is described. Preliminary experimental results both on synthetic and real images are also presented. Ongoing research is aimed at developing a more reliable 


\begin{tabular}{ccccccc}
\hline Image & $R / D$ & $R \hat{j} D$ & $e_{R / D}(\%)$ & $D$ & $\hat{D}$ & $e_{D}(\%)$ \\
\hline Tennis 1 & 0.2 & 0.193 & $3 \%$ & 16 & 17.75 & $10.9 \%$ \\
Tennis 2 & 0.16 & 0.156 & $2.5 \%$ & 20 & 21.39 & $6.9 \%$ \\
Soccer 1 & 0.224 & 0.237 & $5 \%$ & 49 & 47.1 & $5.8 \%$ \\
Soccer 2 & 0.137 & 0.150 & $9.4 \%$ & 80 & 74.67 & $6.6 \%$ \\
\hline
\end{tabular}

Table 2. Localization results for real image. $R / D$ and $D$ is the real measured radiusdistance ratio and the real measured distance (in $\mathrm{cm}$ ); $R \hat{/ D}$ and $\hat{D}$ is the estimated ratio and distance (in $\mathrm{cm}) . e_{R / D}(\%)$ and $e_{D}(\%)$ are the percentage estimation error.

calibration procedure in order to minimize the misalignment between the camera and the mirror and to make the whole procedure more robust to noise.

\section{References}

1. Motilal Agrawal and Larry S. Davis. Camera calibration using spheres: A semidefinite programming approach. In Proceedings of the 9th IEEE International Conference on Computer Vision, page 782, Washington, DC, USA, 2003. IEEE Computer Society.

2. S. Baker and S. K. Nayar. A theory of single-viewpoint catadioptric image formation. International Journal of Computer Vision, 35(2):1 - 22, 1999.

3. Thorsten Bandlow, Michael Klupsch, Robert Hanek, and Thorsten Schmitt. Fast image segmentation, object recognition and localization in a robocup scenario. In RoboCup-99: Robot Soccer World Cup III, volume 1856 of Lecture Notes in Computer Science, pages 174-185, London, UK, 2000. Springer-Verlag.

4. J. Y. Bouguet. Camera calibration toolbox for matlab.

5. J. Bruce, T. Balch, and M. Veloso. Fast and inexpensive color image segmentation for interactive robots. In Proceedings of the IEEE/RSL International Conference on Intelligent Robots and Systems, volume 3, pages 2061-2066, 2000.

6. V. Caglioti and S. Gasparini. On the localization of straight lines in 3d space from single 2d images. In Proceedings of the 2005 Conference on Computer Vision and Pattern Recognition, volume 1, pages 1129-1134, Washington, DC, USA, 2005. IEEE Computer Society.

7. G. Coath and P. Musumeci. Adaptive arc fitting for ball detection in robocup. In APRS Workshop on Digital Image Analysing, 2003.

8. The RoboCup Federation. Robocup official site. http://www.robocup.org.

9. D. Feldman, T. Pajdla, and D. Weinshall. On the epipolar geometry of the crossedslits projection. In Proceedings of the 9th IEEE International Conference on Computer Vision, volume 2, pages 988-995. IEEE, IEEE Computer Society, October 2003.

10. R. Gupta and R. I. Hartley. Linear pushbroom cameras. IEEE Transactions on Pattern Analysis and Machine Intelligence, 19(9):963-975, 1997.

11. K. Kanatani. Geometric computation for machine vision. Oxford University Press, Inc., New York, NY, USA, 1993. 


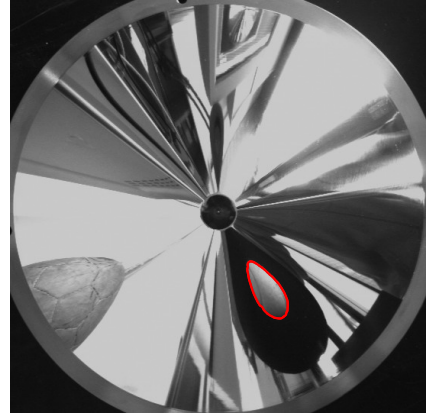

(a)

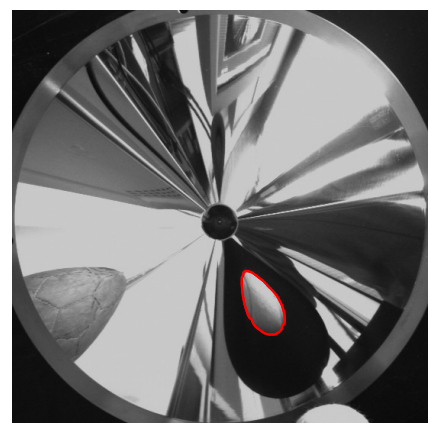

(c)

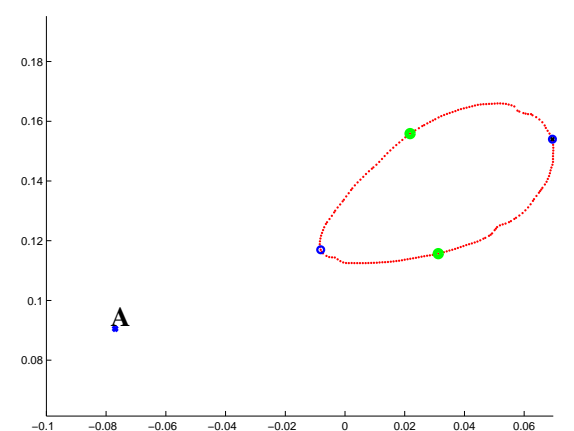

(b)

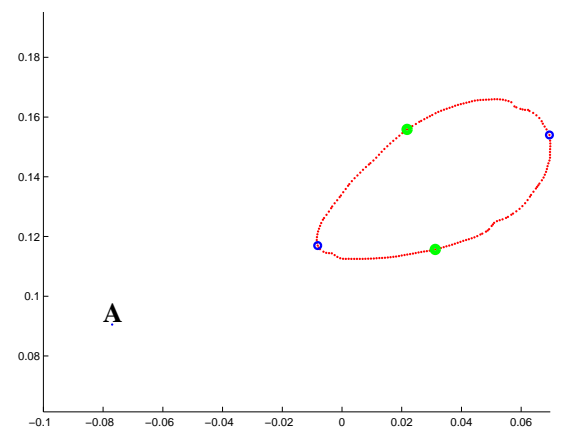

(d)

Fig. 6. Two sample catadioptric images $((\mathrm{a}),(\mathrm{c}))$ of a tennis ball placed at various distances from the mirror axis, and the relevant extracted contour points ((b),(d)) with in evidence the selected pairs of points (circled in green and blue respectively) through which the ball radius and distance is calculated. The point $A$ is the image of the mirror apex.

12. P. Lima, A. Bonarini, C. Machado, F. Marchese, F. Ribeiro, and D. Sorrenti. Omni-directional catadioptric vision for soccer robots. Journal of Robotics and Autonomous Systems, 36:87-102, 2001.

13. S. Mitri, K. Pervlz, H. Surmann, and A. Nchter. Fast color-independent ball detection for mobile robots. In Mechatronics and Robotics, pages 900-905, 2004.

14. S. K. Nayar. Catadioptric omnidirectional camera. In Proceedings of the 1997 Conference on Computer Vision and Pattern Recognition, page 482. IEEE Computer Society, 1997.

15. D. Scaramuzza, S. Pagnottelli, and P. Valigi. Ball detection and predictive ball following based on a stereoscopic vision system. In IEEE International Conference on Robotics and Automation, page 1573, 2005.

16. T. Svoboda, T. Pajdla, and V. Hlaváč. Epipolar geometry for panoramic cameras. In H. Burkhardt and N. Bernd, editors, Proceedings of the 5th European Conference on Computer Vision, volume 1406 of Lecture Notes in Computer Science, pages 218-232, Freiburg, Germany, June 1998. Springer.

17. POV Team. Persistency of vision ray tracer (pov-ray). http: www . povray . org. 


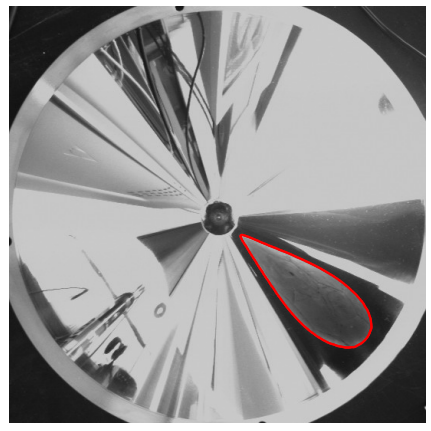

(a)



(c)

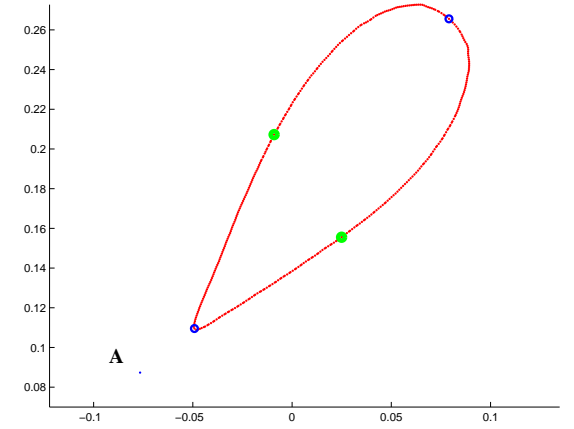

(b)

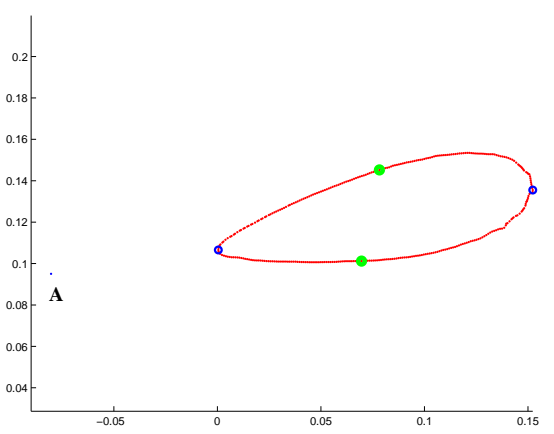

(d)

Fig. 7. Two sample catadioptric images $((\mathrm{a}),(\mathrm{c}))$ of a soccer ball placed at various distances from the mirror axis, and the relevant extracted contour points ((b),(d)) with in evidence the selected pairs of points (circled in green and blue respectively) through which the ball the ball radius and distance is calculated. The point $A$ is the image of the mirror apex.

18. T. Weigel, J.S. Gutmann, M. Dietl, A. Kleiner, and B. Nebel. Cs-freiburg: Coordinating robots for successful soccer playing. IEEE Transaction on Robotics and Automation, 18:685-699, 2002.

19. X. Yingv and H. Zha. Linear catadioptric camera calibration from sphere images. In Proceedings of the International Workshop on Omnidirectional Vision (OMNIVIS 2005), 2005.

20. H. Zhang, G. Zhang, and K.-Y. K. Wong. Camera calibration with spheres: Linear approaches. In International Conference on Image Processing, volume II, pages 1150-1153, Genova, September 2005.

21. Z. Zhang. Flexible camera calibration by viewing a plane from unknown orientations. In Proceedings of the 7th IEEE International Conference on Computer Vision, 1999. 